The Astrophysical Journal, 653: L25-L28, 2006 December 10

(C) 2006. The American Astronomical Society. All rights reserved. Printed in U.S.A.

\title{
ULTRAVIOLET DETECTION OF THE TIDAL DISRUPTION OF A STAR BY A SUPERMASSIVE BLACK HOLE
}

\author{
S. Gezari, ${ }^{1}$ D. C. Martin, ${ }^{1}$ B. Milliard,${ }^{2}$ S. Basa,${ }^{2}$ J. P. Halpern, ${ }^{3}$ K. Forster, ${ }^{1}$ P. G. Friedman,,${ }^{1}$ P. Morrissey, ${ }^{1}$ \\ S. G. NefF, ${ }^{4}$ D. Schiminovich, ${ }^{3}$ M. Seibert, ${ }^{1}$ T. Small, ${ }^{1}$ and T. K. Wyder ${ }^{1}$ \\ Received 2006 August 24; accepted 2006 October 6; published 2006 December 1
}

\begin{abstract}
A supermassive black hole in the nucleus of a galaxy will be revealed when a star passes close enough to be torn apart by tidal forces and a flare of radiation is emitted by the stream of stellar debris that plunges into the black hole. Since common active galactic nuclei have accreting black holes that can also produce flares, a convincing demonstration that a stellar tidal disruption has occurred generally begins with a "normal" galaxy that has no evidence of prior nuclear activity. Here we report a luminous UV flare from an elliptical galaxy at $z=0.37$ in the Groth field of the GALEX Deep Imaging Survey that has no evidence of a Seyfert nucleus from optical spectroscopy and X-ray imaging obtained during the flare. Multiwavelength data collected at the time of the event, and for 2 years following, allow us to constrain, for the first time, the spectral energy distribution of a candidate tidal disruption flare from optical through X-rays. The luminosity and temperature of the radiation and the decay curve of the flare are in excellent agreement with theoretical predictions for the tidal disruption of a star, and provide the strongest empirical evidence for a stellar disruption event to date.
\end{abstract}

Subject headings: galaxies: nuclei — ultraviolet: galaxies

\section{INTRODUCTION}

Dynamical studies of nearby galaxies show that all galaxies with a bulge host a supermassive black hole in their nucleus (Magorrian et al. 1998). Stellar dynamical models (Magorrian \& Tremaine 1999; Wang \& Merritt 2004) predict that once every $10^{4}-10^{5} \mathrm{yr}$ the orbit of a star in the nucleus of a galaxy will pass within the tidal disruption radius of its central supermassive black hole, $R_{T} \approx R_{\star}\left(M_{\mathrm{BH}} / M_{\star}\right)^{1 / 3}$, and the star will be disrupted. A stellar disruption results in a flare of electromagnetic radiation (Frank \& Rees 1976; Lidskii \& Ozernoi 1979 ) as the bound fraction of the stellar debris, $\lesssim 0.5 M_{\star}$ (Rees 1988; Ayal et al. 2000), falls back onto the black hole and is accreted. There is a critical black hole mass above which $R_{T}$ is smaller than the Schwarzschild radius $\left(R_{\mathrm{S}}\right)$, and the star is swallowed whole without disruption (Hills 1975). For a solartype star, $M_{\text {crit }} \sim 10^{8} M_{\odot}$. The luminosity and decay of a tidal disruption flare is dependent on the mass and spin of the central black hole and can be used to directly probe dormant black holes in galaxies for which the sphere of influence of the black hole $\left(R_{\mathrm{sph}}=G M_{\mathrm{BH}} / \sigma_{\star}^{2}\right)$ is unresolved and a dynamical measurement of the black hole mass is not possible.

The start of the flare, $t_{0}$, occurs when the most tightly bound debris first returns to pericenter $\left(R_{p}\right)$ after the star is disrupted at time $t_{D}$, where

$$
\begin{aligned}
\left(t_{0}-t_{D}\right)= & 0.11 k^{-3 / 2}\left(R_{p} / R_{T}\right)^{3}\left(R_{\star} / R_{\odot}\right)^{3 / 2} \\
& \times\left(M_{\star} / M_{\odot}\right)^{-1}\left(M_{\mathrm{BH}} / 10^{6} M_{\odot}\right)^{1 / 2} \mathrm{yr}
\end{aligned}
$$

(Li et al. 2002), and $k=1$ for a star with no spin, and $k=$ 3 for a star that is spun up by tidal interactions to near breakup on disruption. After $t_{0}$, the return rate of the bound debris to pericenter declines as function of time as $\dot{M}(t) \propto[(t-$

\footnotetext{
${ }^{1}$ California Institute of Technology, MC 405-47, 1200 East California Boulevard, Pasadena, CA 91125; suvi@srl.caltech.edu.

${ }^{2}$ Laboratoire d'Astrophysique de Marseille, 13376 Marseille Cedex 12, France.

${ }^{3}$ Department of Astronomy, Columbia University, New York, NY 10027.

${ }^{4}$ Laboratory for Astronomy and Solar Physics, NASA Goddard Space Flight Center, Greenbelt, MD 20771.
}

$\left.\left.t_{D}\right) /\left(t_{0}-t_{D}\right)\right]^{-5 / 3}$ (Evans \& Kochanek 1989), which determines the characteristic power-law decay of the luminosity of the flare over the following months and years. When the mass accretion rate is super-Eddington,

$$
\dot{M}_{\mathrm{Edd}} \equiv L_{\mathrm{Edd}} /\left(\epsilon c^{2}\right)=0.025\left(M_{\mathrm{BH}} / 10^{6} M_{\odot}\right)(\epsilon / 0.1)^{-1} M_{\odot} \mathrm{yr}^{-1},
$$

where $\epsilon$ is the efficiency of converting mass into radiation, the debris is accreted in a thick disk that radiates close to the Eddington luminosity (Ulmer 1999), $L_{\text {Edd }}=1.3 \times$ $10^{44}\left(M_{\mathrm{BH}} / 10^{6} M_{\odot}\right)$ ergs s${ }^{-1}$. The spectrum of a tidal disruption event can thus be characterized by the blackbody temperature of a thick disk radiating at $L_{\text {Edd }}$ at radii ranging from $R_{T}$ to $R_{p}, \quad T_{\text {eff }} \approx\left(L_{\mathrm{Edd}} / 4 \pi \sigma R^{2}\right)^{1 / 4}$, which for a solar-type star is $\sim(2-5) \times 10^{5} \mathrm{~K}$, and peaks in the extreme-UV (Ulmer 1999).

The most unambiguous cases for a stellar disruption occur from host galaxies with no evidence of an active galactic nucleus (AGN) for which an upward fluctuation in the accretion rate could also explain a luminous UV/X-ray flare. A UV flare from the nucleus of the elliptical galaxy NGC 4552 was proposed to be the result of the tidal stripping of a stellar atmosphere (Renzini et al. 1995); however, the possible presence of a persistent, low-luminosity AGN detected in hard X-rays (Xu et al. 2005) makes this interpretation uncertain. The ROSAT All-Sky survey conducted in 1990-1991 sampled hundreds of thousands of galaxies in the soft X-ray band and detected luminous $\left(10^{42}-10^{44} \mathrm{ergs} \mathrm{s}^{-1}\right)$, soft $\left[T_{\mathrm{bb}}=(6-12) \times 10^{5} \mathrm{~K}\right] \mathrm{X}$ ray flares from several galaxies with no previous evidence for AGN activity, and with a flare rate of $\approx 1 \times 10^{-5} \mathrm{yr}^{-1}$ per galaxy (Donley et al. 2002) that is consistent with the theoretical stellar disruption rate. A decade later, follow-up Chandra and XMM-Newton observations of three of the galaxies demonstrated that they had faded by factors of 240-6000, consistent with the $\left(t-t_{D}\right)^{-5 / 3}$ decay of a tidal disruption flare (Komossa et al. 2004; Halpern et al. 2004). Follow-up Hubble Space Telescope (HST) Space Telescope Imaging Spectrograph narrow-slit spectroscopy confirmed two of the galaxies as inactive, qualifying them as the most convincing hosts of a tidal dis- 


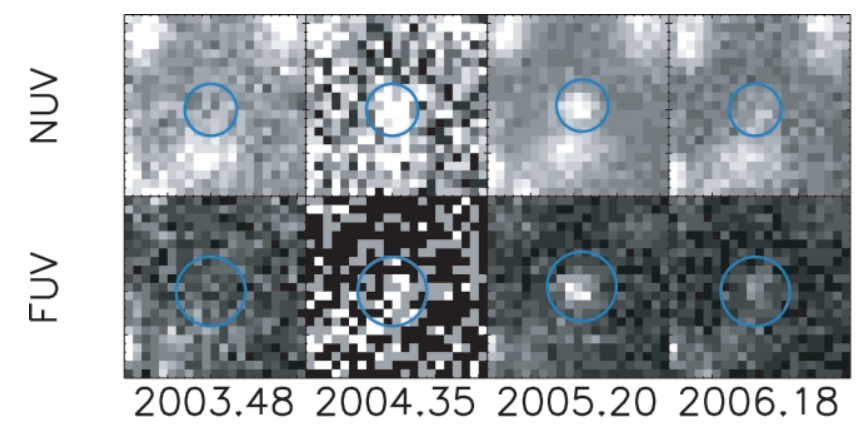

FIG. 1.-GALEX images of the flaring source over 4 years of DIS eclipses coadded into yearly epochs of NUV $\left(t_{\exp }=16,1.5,110\right.$, and $\left.29 \mathrm{ks}\right)$ and FUV $\left(t_{\exp }=16,1.5,41\right.$, and $\left.29 \mathrm{ks}\right)$ observations, labeled with the mean date of each coadd. Blue circles show the $9^{\prime \prime}$ diameter aperture in the NUV, and the $12^{\prime \prime}$ diameter aperture in the FUV used for photometry. No source is detected in NUV and FUV in the 2003.48 epoch. A smaller aperture was used in the NUV to avoid contamination by a pair of foreground-interacting galaxies detected in the NUV just south of the source. The counts from the background in the aperture were determined from the pipeline background image, and an aperture-correction factor of $80 \%$ and $85 \%$ was applied to the NUV and FUV aperture fluxes, respectively, to recover the total energy enclosed in a point source.

ruption event (Gezari et al. 2003). The ROSAT flare with the best-sampled light curve was successfully modeled as the tidal disruption of a brown dwarf or planet (Li et al. 2002), although its host galaxy was subsequently found to have a low-luminosity Seyfert nucleus (Gezari et al. 2003).

\section{OBSERVATIONS}

We initiated a program to take advantage of the UV sensitivity, large volume, and temporal sampling of the Galaxy Evolution Explorer (GALEX) Deep Imaging Survey (DIS) to search for stellar disruptions in the nuclei of galaxies over a large range of redshifts. The DIS covers $80 \mathrm{deg}^{2}$ of sky in the farultraviolet (FUV; $\lambda=1344-1786 \AA$ ) and near-ultraviolet (NUV; $\lambda=1771-2831 \AA$ ) with a total exposure time of 30 $150 \mathrm{ks}$, that is accumulated in $\sim 1.5 \mathrm{ks}$ eclipses (when the satellite's 98.6 minute orbit is in the shadow of the Earth). Due to target visibility and mission planning constraints, some DIS fields are observed over a baseline of 2-4 years to complete the total exposure time. This large range in cadence of the observations allows us to probe variability on timescales from hours to years. Here we present a UV flare discovered in the GALEX $1.2 \mathrm{deg}^{2}$ Groth field at a position of right ascension $14^{\mathrm{h}} 19^{\mathrm{m}} 29^{\mathrm{s}} .8$, declination $+52^{\circ} 52^{\prime} 6^{\prime \prime}$. We use multiwavelength survey data from the All-Wavelength Extended Groth Strip International Survey (AEGIS; Davis et al. 2006) and the Canada-France-Hawaii Telescope Legacy Survey (CFHTLS) to identify the host of the flare and study the broadband properties of the flare and its subsequent decay. Throughout this Letter, calculations are made using Wilkinson Microwave Anisotropy Probe cosmological parameters (Bennett et al. 2003): $H_{0}=$ $71 \mathrm{~km} \mathrm{~s}^{-1} \mathrm{Mpc}^{-1}, \Omega_{M}=0.27$, and $\Omega_{\Lambda}=0.73$.

The UV flare reported here was discovered as a result of its large amplitude of variability during 4 years of DIS observations. Figure 1 shows the discovery images in the NUV and FUV of the source, with the eclipses co-added into yearly epochs, and Figure 2 shows its detailed NUV+FUV light curve. The source is undetected in the NUV and FUV in 2003 June 21-29 and then appears in 2004 March 25-June 24, indicating an amplitude of variability of $\Delta m \gtrsim 2 \mathrm{mag}$. The source then decays monotonically by $\sim 2.0$ mag over 2 years to almost

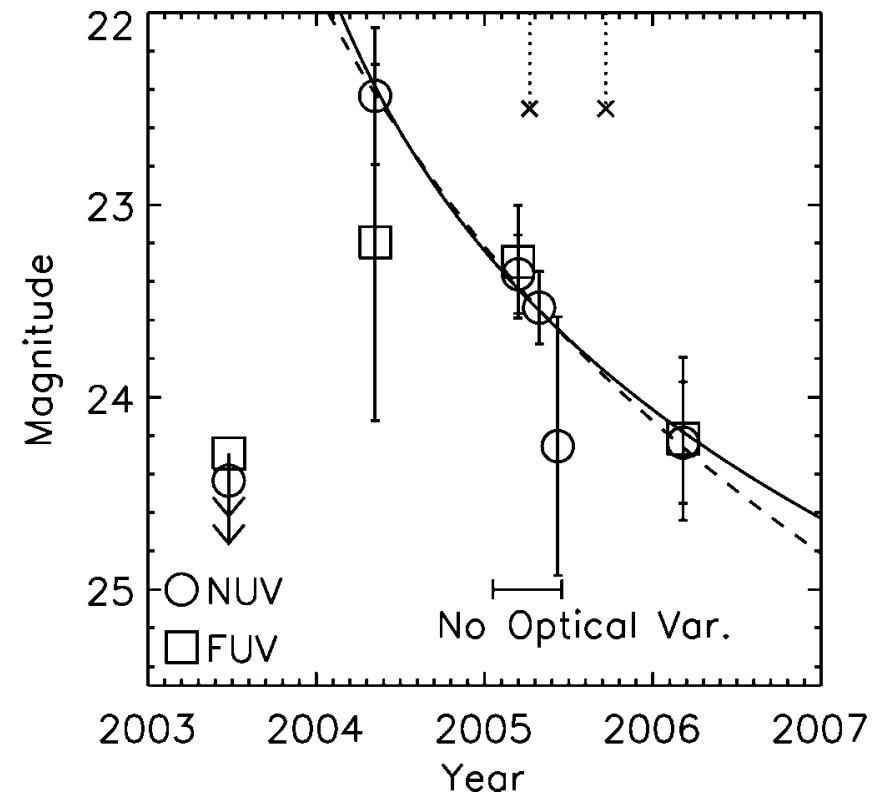

FIG. 2.-GALEX light curve in the FUV $\left(\lambda_{\text {eff }}=1528 \AA\right)$ and NUV $\left(\lambda_{\text {eff }}=2271 \AA\right)$. Error bars show $68 \%$ confidence intervals, and arrows show $95 \%$ confidence upper limits based on Bayesian statistics. For the later observations in 2005 the FUV detector was temporarily not operational. The least-squares fit to the NUV light curve with a $\left(t-t_{D}\right)^{-5 / 3}$ decay is shown with a thick solid line, yielding a best-fit time of disruption $t_{D}=2003.3$, with a $1 \sigma$ error of 0.2 yr. The least-squares fit to the NUV light curve with the power-law index, $\left(t-t_{D}\right)^{-n}$, and $t_{D}$ allowed to vary is shown with a dashed line, with $t_{D}=2002 \pm 2$ and $n=3 \pm 2$. Dotted lines with a cross indicate times of two sets of $100 \mathrm{ks}$ Chandra $0.3-10 \mathrm{keV}$ observations. An extremely soft X-ray source was detected during the first set observations, and no source was detected in the second set of observations. CFHTLS monitoring data from 2005 January to June in $g\left(\lambda_{\text {eff }}=4763 \AA\right), r\left(\lambda_{\text {eff }}=6174 \AA\right), i\left(\lambda_{\text {eff }}=\right.$ $7619 \AA)$, and $z\left(\lambda_{\text {eff }}=8847 \AA\right)$ with $m_{\text {lim }}=25$, detect no variable optical source during the UV flare.

below the detection threshold in 2006 March 5-7. Archival AEGIS $50 \mathrm{ks}$ Chandra $(0.3-10 \mathrm{keV})$ observations on 2005 April 6 and 7 detected an extremely soft X-ray source that appeared on the second day of the observations, with 10 photons with energies between 0.3 and $0.8 \mathrm{keV}$, indicating a spectral slope, $f_{E} \propto E^{-\Gamma}$, of $\Gamma=7 \pm 2$ (reduced $\chi^{2}=0.92$ ), for a column density of neutral hydrogen fixed to the Galactic value. No source was detected in the following $50 \mathrm{ks}$ Chandra observations on 2005 September 20 and 23.

\section{INTERPRETATION}

The UV flare and variable soft X-ray source are coincident with an elliptical galaxy at $z=0.3698$ (luminosity distance, $d_{L}=1970 \mathrm{Mpc}$ ). Figure 3 shows the AEGIS HST ACS optical image with the UV and X-ray positions overplotted, and the AEGIS Keck DEIMOS optical spectrum from the DEEP2 survey of the host galaxy taken on 2005 March 9. The spectrum has strong stellar absorption features typical of an early-type galaxy. We marginally detect faint narrow [O III] $\lambda 5007$ line emission with $L([\mathrm{O} \mathrm{III}])=(8 \pm 4) \times 10^{39} \mathrm{ergs} \mathrm{s}^{-1}$. The nondetection of a hard X-ray source by Chandra places an upper limit to the 3-20 keV luminosity of a power-law AGN spectrum in the nucleus of $L_{\mathrm{X}} \lesssim 1 \times 10^{42} \mathrm{ergs} \mathrm{s}^{-1}$. The correlation between $L([\mathrm{O} \mathrm{III}])$ and $L_{\mathrm{X}}(3-20 \mathrm{keV})$ observed for local AGNs (Heckman et al. 2005) predicts $L([\mathrm{O} \mathrm{III}])<10^{39} \mathrm{erg} \mathrm{s}^{-1}$, which suggests that the possible [O III] line emission has too high an $L([\mathrm{O} \mathrm{III}]) / L_{\mathrm{X}}$ ratio to be associated with AGN activity and may be from low levels of star formation. We obtained a follow- 


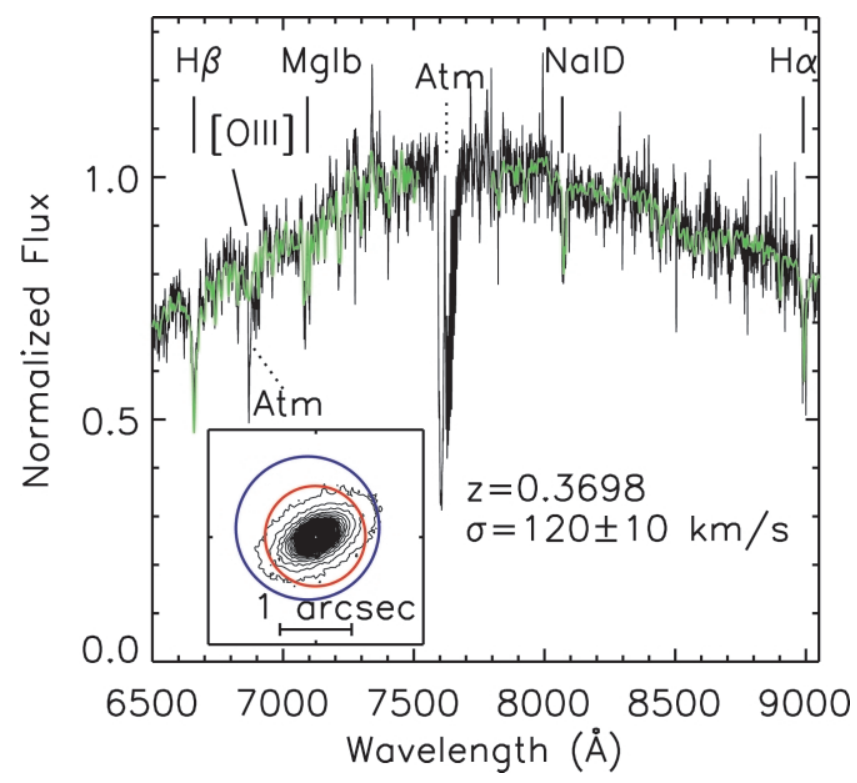

FIG. 3.-Inset: AEGIS HST ACS I-band image $\left(\lambda_{\text {eff }}=8140 \AA\right)$ of the galaxy host with the GALEX 1" position error circle (blue) of the UV flare and the Chandra 0.7 position error circle (red) of the soft X-ray source, with a 1" systematic astrometry correction applied to the Chandra position measured from the shift of the Chandra astrometry with respect to ACS for a nearby star. The morphology of the galaxy is determined from a two-dimensional bulge/disk decomposition of the ACS I-band image (Simard 1998), which results in a bulge fraction of 0.72 , and a half-light radius of the bulge, $R_{e}=0 " 43$. Spectrum: AEGIS Keck DEIMOS spectrum from the DEEP2 survey of the galaxy host obtained on 2005 March 9, smoothed by 6 pixels $(\sim 2 \AA)$, and not corrected for the instrumental response. The spectrum shows strong stellar absorption lines typical of an early-type galaxy, with a marginal detection of narrow [O III] $\lambda 5007$ line emission. The best-fitting BruzualCharlot early-type galaxy template (Bruzual \& Charlot 2003) scaled to the continuum of the spectrum is shown in green. The luminosity-weighted line of sight velocity dispersion measured using stellar templates Moran et al. 2005 within the $1^{\prime \prime}$ slit width of DEIMOS is $120 \pm 10 \mathrm{~km} \mathrm{~s}^{-1}$, which corresponds to an aperture of $1.2 R_{e}$, for which the correction to an aperture of $R_{e}$ is $<5 \%$ (Gebhardt et al. 2000).

up spectrum after the flare discovery on 2006 June 1 with the Double Spectrograph on the Palomar 200 inch telescope and again detected no strong emission lines. CFHTLS variability monitoring data from 2005 January to June show no detection of a variable optical source in the galaxy, with up to five observations per month with a sensitivity of $m_{\mathrm{lim}}=25$.

The lack of variable optical emission during the UV flare puts an important constraint on its nature. Supernovae ( $\mathrm{SNe}$ ) have light curves that extend over months, similar to the flare observed here, but their emission peaks at optical wavelengths and is intrinsically faint shortward of $\sim 3000 \AA$ (Panagia 2003; Brown et al. 2005). Gamma-ray bursts (GRBs) have powerlaw decays, but again, their emission is brighter at optical wavelengths (Mészáros \& Rees 1997). The characteristic power-law continuum of AGN emission extends from optical to extremeUV as $f_{\nu} \propto \nu^{-\alpha}$, where $0.43 \leq \alpha \leq 2$ (Zheng et al. 1997; Dietrich et al. 2002). If the UV flare were an upward fluctuation of AGN continuum emission, a power-law spectrum with $\alpha \geq 0.43$ would produce an optical source with $m<23 \mathrm{mag}$ during the CFHTLS observations and power a broad $\mathrm{H} \beta$ line with a luminosity of $\geq 1 \times 10^{41} \mathrm{ergs} \mathrm{s}^{-1}$ (Dietrich et al. 2002) at the time of the Keck spectrum, both of which are definitively ruled out by the observations. In summary, the flare is most probably not due to AGN activity because it displays (1) no optical variability, (2) no broad emission lines, (3) a soft Xray spectrum with no hard X-ray component, and (4) an early-

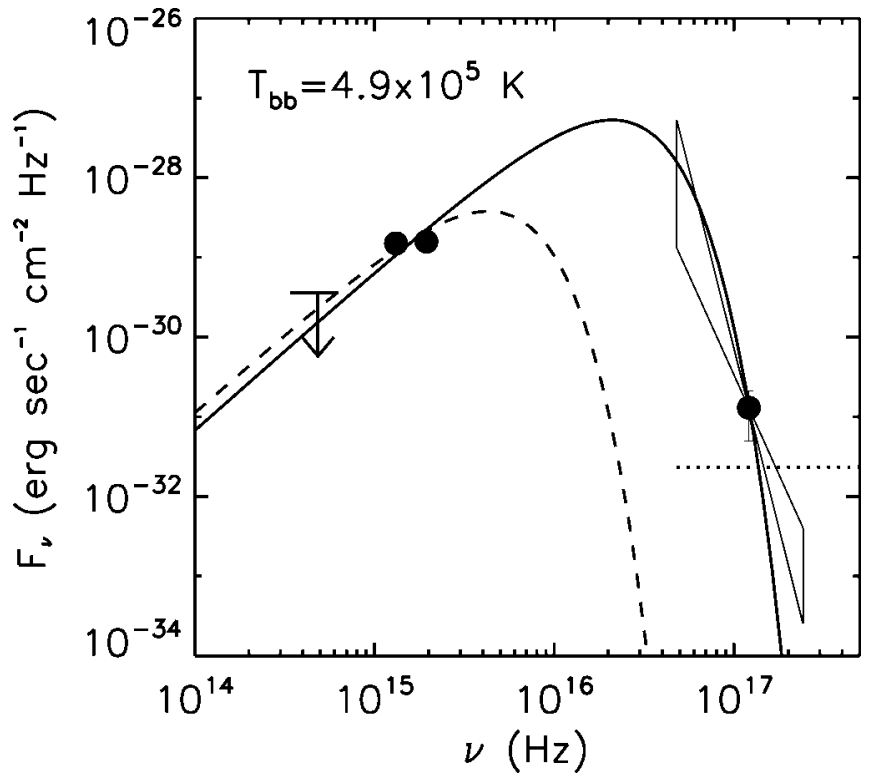

FIG. 4.-Spectral energy distribution from optical to X-rays of the flare on 2005 April 7, with the best blackbody fit with $T_{\mathrm{bb}}=4.9 \times 10^{5} \mathrm{~K}$ shown with a solid line. The spectral slope of the extremely soft X-ray detection, $f_{\nu} \propto$ $E^{-\Gamma}$, of $\Gamma=7 \pm 2$ is also shown, corresponding to an flux density at $0.5 \mathrm{keV}$ of $(1.3 \pm 0.8) \times 10^{-31} \mathrm{erg} \mathrm{s}^{-1} \mathrm{~cm}^{-2} \mathrm{~Hz}^{-1}$, a factor of $\sim 4$ above the detection limit of Chandra (dotted line). Since the X-ray flux appears above the detection threshold of Chandra for only part of the observations, the blackbody fit gives an upper limit to $T_{\mathrm{bb}}$ during the flare. Dashed line shows the lowest temperature blackbody consistent with the UV flux densities and optical upper limits, with $T_{\mathrm{bb}}=1 \times 10^{5} \mathrm{~K}$.

type galaxy spectrum with possible narrow [O III] emission with a high $[\mathrm{O} \mathrm{III}] / L_{\mathrm{X}}$ ratio characteristic of star formation. In addition, an SN or GRB origin is unlikely because of the high UV/optical ratio.

The spectral energy distribution (SED) of the flare from optical to X-rays on 2005 April 7 is shown in Figure 4. A blackbody fit to the SED is strongly constrained by the soft $\mathrm{X}$-ray flux density, which is best fit for a rest-frame $T_{\mathrm{bb}}=$ $4.9 \times 10^{5} \mathrm{~K}$, shown with a solid line in Figure 4, which for $L_{\nu_{e}=(1+z) \nu_{o}}=f_{v_{o}}\left(4 \pi d_{L}^{2}\right) /(1+z)$, corresponds to $R_{\mathrm{bb}}=6.2 \times$ $10^{12} \mathrm{~cm}$ and $L_{\mathrm{bol}}=1.2 \times 10^{45} \mathrm{ergs} \mathrm{s}^{-1}$. The steep Wein's tail of the blackbody curve is consistent with the extremely soft spectral slope of the X-ray source. However, since the X-ray flux varies on a short timescale and only appears above the detection threshold for part of the observations, we consider this fit an upper limit to $T_{\mathrm{bb}}$ for the duration of the flare. The lowest $T_{\mathrm{bb}}$ that is consistent with the UV flux densities and the optical upper limits is $\sim 1 \times 10^{5} \mathrm{~K}$, shown with a dashed line in Figure 4, which corresponds to $R_{\mathrm{bb}}=1.6 \times 10^{13} \mathrm{~cm}$ and $L_{\text {bol }}=1.3 \times 10^{43} \mathrm{ergs} \mathrm{s}^{-1}$. This range of temperatures and luminosities is in excellent agreement with the theoretical predictions for a tidal disruption flare.

\section{DISCUSSION}

The characteristic tidal disruption $\left(t-t_{D}\right)^{-5 / 3}$ power-law decay fit to the UV light curve yields a best-fit time of disruption of $t_{D}=2003.3 \pm 0.2$. If we allow the power-law index of the decay, $\left(t-t_{D}\right)^{-n}$, to vary as well as $t_{D}$, we get a comparable fit, but with much less constrained values for $t_{D}=2002 \pm 2$ and $n=3 \pm 2$. The GALEX observations do not cover the rise of the UV flux to its peak value; however, we can constrain the time of the peak $\left(t_{0}\right)$ to be between the time of the GALEX detection beginning on 2004 March 25 and the latest date of 
the nondetection on 2003 June $29,\left(t_{0}-t_{D}\right)=0.2( \pm 0.2)-$ $0.9( \pm 0.2)$ yr. We estimate the total energy released during the flare by integrating the best-fit $\left(t-t_{D}\right)^{-5 / 3}$ light curve from the time of the peak of the flare to infinity, $E_{\text {tot }}=\int_{t_{0}}^{\infty} L(t) d t$. If on 2005 April $6-7,1.3 \times 10^{43} \mathrm{ergs} \mathrm{s}^{-1} \lessgtr L_{\text {bol }} \leqslant 1.2 \times$ $10^{45} \mathrm{ergs} \mathrm{s}^{-1}$, and $\left(t_{0}-t_{D}\right) \lesssim 1.1 \mathrm{yr}$, this yields a minimum total energy released of $1.8 \times 10^{51} \mathrm{ergs} \lessgtr E_{\text {tot }} \lesssim 1.7 \times 10^{53}$ ergs, and a minimum mass accreted, $M_{\mathrm{acc}}=E_{\mathrm{tot}} /\left(\epsilon c^{2}\right)$, of $0.01 \quad M_{\odot}(\epsilon / 0.1)^{-1} \lesssim M_{\text {acc }} \lesssim 0.9 \quad M_{\odot}(\epsilon / 0.1)^{-1}, \quad$ assuming $T_{\mathrm{bb}}=(1-5) \times 10^{5} \mathrm{~K}$ during the flare.

For a solar-type star, the limits on $\left(t_{0}-t_{D}\right)$ imply $k^{3} 3 \times$ $10^{6} M_{\odot} \lesssim M_{\mathrm{BH}} \lesssim k^{3} 7 \times 10^{7} M_{\odot}$, where $k$ depends on the spin-up of the star, with a hard upper limit on the black hole mass of $M_{\mathrm{BH}}<M_{\text {crit }}=1.15 \times 10^{8}\left(r^{3} / m\right)^{1 / 2} M_{\odot}$, where $r=$ $R_{\star} / R_{\odot}$, and $m=M_{\star} / M_{\odot}$, in order to satisfy the condition that $R_{T}>R_{\mathrm{S}}$, and a stellar disruption can occur. The radius of the flare emission also places constraints on $M_{\mathrm{BH}}$. The debris disk should form within the tidal disruption radius of the central black hole. The blackbody fits to the flare SED have $R_{\mathrm{bb}} \lesssim 1.6 \times 10^{13} \mathrm{~cm}$ which, if $R_{\mathrm{bb}} \lesssim R_{T}$, places a lower limit on the black hole mass of $M_{\mathrm{BH}} \gtrsim 1.2 \times 10^{7} M_{\odot}$. The radius of the emission should also be greater than the minimum stable particle orbit for the black hole $\left(R_{\mathrm{ms}}\right)$, which ranges from $R_{\mathrm{ms}}=6 R_{g}$ for a black hole with no spin, down to $R_{\mathrm{ms}}=R_{g}$ for a maximally spinning black hole, where $R_{g}=G M_{\mathrm{BH}} / c^{c^{g}}$. For $R_{\mathrm{bb}}>R_{\mathrm{ms}}$, this requires that $M_{\mathrm{BH}}<1.1 \times 10^{8} M_{\odot}$ and is consistent with $M_{\mathrm{BH}}<M_{\text {crit }}$ for a solar-type star. We can also use the strong correlation between black hole mass and stellar velocity dispersion established for local galaxies (Tremaine et al. 2002), $\log \left(M_{\mathrm{BH}} / M_{\odot}\right) \sim 8.13+4.02 \log \left(\sigma_{e} / 200 \mathrm{~km} \mathrm{~s}^{-1}\right)$, to estimate $M_{\mathrm{BH}}$. The stellar velocity dispersion within the halflight radius $\left(R_{e}\right)$ of the bulge of the galaxy, $\sigma_{e}=120 \pm 10 \mathrm{~km}$ $\mathrm{s}^{-1}$, yields a mass of $2_{-1}^{+2} \times 10^{7} M_{\odot}$, where the $1 \sigma$ error includes the intrisic scatter of the correlation of $\sim 0.3$ dex.

To summarize, the black hole mass estimated from the $M_{\mathrm{BH}^{-}} \sigma$ relation $\left(M_{\mathrm{BH}}=2_{-1}^{+2} \times 10^{7} M_{\odot}\right)$ is consistent with the constraints on the black hole mass from the blackbody spectrum such that $R_{\mathrm{ms}}<R_{\mathrm{bb}} \lesssim R_{T}\left(1.2 \times 10^{7} M_{\odot} \lesssim M_{\mathrm{BH}}<\right.$ $1.1 \times 10^{8} M_{\odot}$ ), with the limits derived from the time delay between the time of the disruption and the peak of the flare $\left(k^{3} 3 \times 10^{6} M_{\odot} \lesssim M_{\mathrm{BH}} \lesssim k^{3} 7 \times 10^{7} M_{\odot}\right)$, and with the con- dition that $M_{\mathrm{BH}}<M_{\text {crit }}=1.15 \times 10^{8}$ for a solar-type star to be disrupted. It is encouraging that these various independent black hole mass estimates are in agreement with each other as long as $k<3$, and the star was not spun up to near breakup on disruption. The GALEX DIS has proven to be a promising survey for detecting stellar disruption events so far. With a larger sample of tidal disruption flares, we can begin to measure the distribution of masses and spins of black holes in the nuclei of normal galaxies as a function of redshift, unbiased by the minority of galaxies that are AGNs.

We thank the anonymous referee for helpful comments, including the suggestion to add a discussion of the radius of the flare emission. We thank C. L. Slesnick for carrying out our target-of-opportunity observation with the Double Spectrograph on the Palomar 200 inch telescope, V. Villar for the twodimensional bulge/disk composition of the AEGIS HST ACS image, and S. M. Moran for measurement of the stellar velocity dispersion of the AEGIS DEEP2 DEIMOS spectrum. S. G. was supported in part by the Volontariat International-CNES of France, and through Chandra Grant Award G06-7099X issued by the Chandra X-ray Observatory, which is operated by the SAO for and on behalf of NASA. We gratefully acknowledge NASA's support for construction, operation, and science analysis for the GALEX mission, developed in cooperation with CNES and the Korean Ministry of Science and Technology. The AEGIS collaboration acknowledges support from the NASA/ESA HST grant GO-10134 for the Extended Groth Strip observations, obtained at STScI, which is operated by AURA, Inc., under a NASA contract. The AEGIS collaboration also acknowledges support from the NSF grant AST 05-07483 for the DEEP2 survey observations with DEIMOS at the W. M. Keck Observatory. This work is based on observations obtained with MegaPrime/MegaCam, a joint project of CFHT and CEA/ DAPNIA, at the CFHT which is operated by the NRC of Canada, the CNRS of France, and the University of Hawaii. This work is also based in part on data products produced at TERAPIX and the Canadian Astronomy Data Centre as part of the CFHT Legacy Survey, a collaborative project of NRC and CNRS.

\section{REFERENCES}

Ayal, S., Livio, M., \& Piran, T. 2000, ApJ, 545, 772

Bennett, C. L., et al. 2003, ApJS, 148, 1

Brown, P. J., et al. 2005, ApJ, 635, 1192

Bruzual, G., \& Charlot, S. 2003, MNRAS, 344, 1000

Davis, M., et al. 2006, ApJ, submitted

Dietrich, M., et al. 2002, ApJ, 581, 912

Donley, J. L., Brandt, W. N., Eracleous, M. J., \& Boller, Th. 2002, AJ, 124, 1308

Evans, C. R., \& Kochanek, C. S. 1989, ApJ, 346, L13

Frank, J., \& Rees, M. J. 1976, MNRAS, 176, 633

Gebhardt, K., et al. 2000, ApJ, 539, L13

Gezari, S., Halpern, J. P., Komossa, S., Grupe, D., \& Leighly, K. M. 2003, ApJ, 592, 42

Halpern, J. P., Gezari, S., \& Komossa, S. 2004, ApJ, 604, 572

Heckman, T. M., Ptack, A., Hornschemeier, A., \& Kauffmann, G. 2005, ApJ, 634,161

Hills, J. G. 1975, Nature, 254, 295

Komossa, S., et al. 2004, ApJ, 603, L17
Li, L-X., Narayan, R., \& Menou, K. 2002, ApJ, 576, 753

Lidskii, V. V., \& Ozernoi, L. M. 1979, Soviet Astron. Lett., 5, 16

Magorrian, J., \& Tremaine, S. 1999, MNRAS, 309, 447

Magorrian, J., et al. 1998, AJ, 115, 2285

Mészáros, P., \& Rees, M. J. 1997, ApJ, 476, 232

Moran, S. M., et al. 2005, ApJ, 634, 977

Panagia, N. 2003, in Supernovae and Gamma-Ray Bursters, ed. K. Weiler \& A. F. M. Moorwood (Baltimore: STScI), 113

Rees, M. J. 1988, Nature, 333, 523

Renzini, A., et al. 1995, Nature, 378, 39

Simard, L. 1998, in ASP Conf. Ser. 145, Astronomical Data Analysis Software Systems VII, ed. R., Albrecht, R. N. Hook \& H. A. Bushouse (San Francisco: ASP), 108

Tremaine, S., et al. 2002, ApJ, 574, 740

Ulmer, A. 1999, ApJ, 514, 180

Wang, J., \& Merritt, D. 2004, ApJ, 600, 149

$\mathrm{Xu}$, Y., et al. 2005, ApJ, 631, 809

Zheng, W., et al. 1997, ApJ, 475, 469 